\title{
Multicast-Based Inference of Network-Internal Loss Performance
}

\author{
Hui Tian and Hong Shen \\ Graduate School of Information Science \\ Japan Advanced Institute of Science and Technology \\ 1-1 Asahidai, Tatsunokuchi, Ishikawa, 923-1292, Japan \\ hui-t,shen@jaist.ac.jp
}

\begin{abstract}
The use of multicast traffic as measurement probes is efficient and effective to infer network-internal characteristics. We propose a new statistical approach to infer network internal link loss performance from end-to-end measurements. Incorporating with the procedure of topology inference, we present an inference algorithm that can infer loss rates of individual links in the network when it infers the network topology. It is proved that the loss rate inferred by our approach is consistent with the real loss rate as the number of probe packets tends to infinity. The approach is also extended to general trees case for loss performance inference. Loss rate-based scheme on topology inference is built in view of correct convergence to the true topology for general trees.
\end{abstract}

Key words: Multicast network, loss rate, inference.

\section{Introduction}

How to measure network performance accurately plays an important role on the successful design, control and management of networks. The existing approaches to evaluating network performance are classified into three types: (i) collecting statistics at internal nodes and using network management packages to generate link-level performance reports; (ii) characterizing network performance based on end-to-end behavior of point-to-point traffic such as that generated by TCP or UDP; (iii) inferring link-level loss behavior through multicast probe traffic as end-to-end measurements.
The first approach requires the authorized access to a wide range of internal nodes in an administratively diverse network which can hardly be realized in practice. Introducing new measurement mechanisms into the nodes themselves is likewise difficult because it requires persuading large companies to alter their products. Also, the composition of many such small measurements to form a picture of end-to-end performance is not completely understood.

The second approach using the prevalent protocol can collect link-level statistics from end-to-end point-to-point measurements. It is, however, very costly to perform such kind of unicast probe in a large-scale network.

Link-level loss and delay inference from multicast endto-end measurements has recently been proposed as an efficient approach to analyze network performance such as $[1,2,3,7,8]$. The key idea underlying the third approach is that multicast traffic introduces correlation in the end-to-end measurements at receivers. This correlation can, in turn, be used to infer internal network characteristics such as link loss and link delay. The approach we propose, which is mainly focused on loss performance inference, inherits the advantage of the third approach. Compared with the principal analytical tool MLEs used in [1,3], our approach is simpler and more efficient.

Incorporating the topology inference algorithm we recently proposed in [9], we present an algorithm to estimate the loss rate of all the individual links in the multicast network using only statistical loss observations at receivers. We also extend this approach to the general case. It is observed that, based on the information of link loss rate, the quality of multicast topology inference can be improved. 
The paper is organized as follows. In Section 2 the mathematical loss model of multicast network is described. Section 3 derives the estimation method for loss rate on internal links and proposes an algorithm that can infer topology and loss performance simultaneously. Extension of loss inference to the general tree is discussed in Section 4, also a scheme of topology inference based on loss inference is given. Section 5 concludes the paper.

\section{Description of The Loss Model}

A physical multicast tree is represented by a tree model comprising actual network elements (the nodes) and communication links connecting them as cited in $[6,4,5]$. The multicast tree model is denoted by $T=(V, L)$ with node set $V$, including the source node 0 and receiver set $R \subset V$ and link set $L$. A link is said to be internal if neither of its endpoints is the root or a leaf node. Each non-leaf node $k$ has a set of children node $d(k)=\left\{d_{i}(k) \mid 1 \leq i \leq n_{k}\right\}$, and each non-root node $k$ has a parent $p(k)$. The link $(p(k), k) \in L$ is denoted by link $k$. Let $a(U)$ denote the nearest common ancestor of a node set $U \subset V$. Nodes in $U$ are said to be siblings if they have the same parent. The subtree of $T$ rooted at $k$ is denoted by $T(k)=(V(k), L(k))$, and the receiver set $R(k)=R \cap V(k)$.

For each link an independent Bernoulli loss model is assumed with each probing packet being successfully transmitted across link $k$ with probability $p_{k}$. Thus the progress of each probing packet down the tree is described by an independent copy of a stochastic process $X=\left(X_{k}\right)_{k \in V}$ as follows. $X_{0}=1, X_{k}=1$ if the probing packet reaches node $k \in V$ and 0 otherwise. If $X_{k}=0, X_{j}=$ $0, \forall j \in d(k)$. Otherwise, $P\left[X_{j}=1 \mid X_{k}=1\right]=p_{j}$ and $P\left[X_{j}=0 \mid X_{k}=1\right]=1-p_{j}=\alpha_{j}$, where $\alpha_{j}$ denotes the probability of a probe is lost on link $j$. Define $p_{0}=1$. The pair $(T, p)$ is called a loss tree. $P_{T, p}$ denotes the distribution of $X$ on the loss tree $(T, p) . X_{k}^{(i)}$ denotes the loss measurement of node $k$ for $i$ th probe packet. Thus for $n$ probe packets, the $0-1$ sequence maintained on the node $k$ is denoted by $\left\{X^{(n)}(k)\right\}, k \in V$.

\section{Inference on Link-level Loss Performance}

Multicast network in binary tree form is firstly considered for simplicity. From the " $0-1$ " sequences observed at receivers, we can infer the sequence maintained by internal nodes in bottom up fashion. The approach to inferring the link-level loss performance is proposed in the section based on all the " $0-1$ " sequences.

\subsection{Approach to loss performance inference}

If a probe packet reaches any node descended from node $i$, it certainly reaches the node $i$. So we can simply deduce the sequence of internal nodes by the Equation (1).

$$
X^{(n)}(i)=\vee_{l \in R(i)} X^{(n)}(l)
$$

Among the sequence of a node $i$, the component of 0 means the correspondent probe packet is lost in the path from the root to the node $i$. Comparing the sequence of the node $i$ with that of its siblings $j$, if $j$ receives a probe packet, but the node $i$ hasn't received, we consider the probe packet is lost in the link $i$. Thus, those components of 0 in the sequence of the node $i$ can be determined to be caused by loss in link $i$ if the correspondent components in the sequence of node $j$ is 1 . Among those components whose correspondent value of both the node $i$ and $j$ are 0 , some are lost in the path from the root to their parent node, others are lost in both the links directly connecting the siblings at the same time. We suppose $\gamma$ to be the ratio of the number of 0 components in the sequence caused by both links meanwhile other than by their common links to the total number of 0 components.

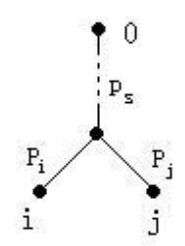

\section{Figure 1. A Transmission Model}

The probability of a probe packet transmitted through the link $i$ successfully can be estimated as the ratio of the number of accepted probe packets at the node $i$ to the number of accepted probe packets at its immediate parent node. According to the above discussion, it can be denoted as the following equations.

$$
\hat{p}_{i}=\frac{n_{i}^{1}}{n_{i}^{1}+n_{j}^{1}-n_{i j}^{1}+\gamma \cdot n_{i j}^{0}}
$$




$$
\begin{aligned}
& =\sum_{k=1}^{n} X_{i}^{(k)} \mid\left[\sum_{k=1}^{n} X_{i}^{(k)}+\sum_{k=1}^{n} X_{j}^{(k)}-\sum_{k=1}^{n} X_{i}^{(k)} \wedge X_{j}^{(k)}\right. \\
& \left.+\gamma \cdot\left(n-\sum_{k=1}^{n} X_{i}^{(k)} \vee X_{j}^{(k)}\right)\right]
\end{aligned}
$$

Where $n_{i}^{1}$ denotes the number of probe packets transmitted trough link $i$ successfully, and $n_{i}^{0}$ denotes the number of lost probe packets on link $i . n_{i j}^{1}$ is denoted the number of probe packets transmitted successfully on both link $i$ and $j$ at the same time. Likewise, $n_{i j}^{0}$ means the number of probe packets lost on both link $i$ and $j$ at the same time.

Assume $p_{s}$ to represent the probability of transmitting probe packets successfully from the root to the sibling's parent node as shown in Figure 1.

Firstly, we give the lemma to estimate $p_{s}$ on which calculation of the loss rate on link $i$ by the Lemma 2 is based.

Lemma $1 p_{s}$ can be estimated by the following formula

$$
\hat{p}_{s}=\frac{n_{i}^{1} \cdot n_{j}^{1}}{n \cdot n_{i j}^{1}}
$$

\section{Proof}

We have known $A(i, j)$ is estimated by $A^{(n)}(i, j)$ as mentioned in[5].

$A^{(n)}(i, j)=\frac{\sum_{m=1}^{n} X^{(m)}(i) \cdot \sum_{m=1}^{n} X^{(m)}(j)}{n \cdot \sum_{m=1}^{n} X^{(m)}(i) \cdot X^{(m)}(j)}=\frac{n_{i}^{1} \cdot n_{j}^{1}}{n \cdot n_{i j}^{1}}$

Because,

$$
\begin{gathered}
\lim _{n \rightarrow \infty} n_{i}^{1} / n=p_{s} \cdot p_{i} \\
\lim _{n \rightarrow \infty} n_{j}^{1} / n=p_{s} \cdot p_{j} \\
\lim _{n \rightarrow \infty} n_{i j}^{1} / n=p_{s} \cdot p_{i} \cdot p_{j}
\end{gathered}
$$

We have,

$$
\lim _{n \rightarrow \infty} A^{(n)}(i, j)=\lim _{n \rightarrow \infty} \frac{n_{i}^{1} \cdot n_{j}^{1}}{n \cdot n_{i j}^{1}}=p_{s}
$$

Therefore, with finite probe packets we can use $\frac{n_{i}^{1} \cdot n_{j}^{1}}{n \cdot n_{i j}^{1}}$ to estimate $p_{s} . \sharp$

Lemma 2 If node $i$ and $j$ are siblings in the real multicast network, the loss rate on link $i$ and $j$ denoted by $\hat{\alpha}_{i}$ and $\hat{\alpha}_{j}$ respectively can be estimated on both of their " $0-1$ " sequences.

$$
\hat{\alpha}_{i}=1-\hat{p}_{i}=1-\frac{\sum_{k=1}^{n} X_{i}^{(k)} \wedge X_{j}^{(k)}}{\sum_{k=1}^{n} X_{j}^{(k)}}
$$

$$
\hat{\alpha}_{j}=1-\hat{p}_{j}=1-\frac{\sum_{k=1}^{n} X_{i}^{(k)} \wedge X_{j}^{(k)}}{\sum_{k=1}^{n} X_{i}^{(k)}}
$$

\section{Proof}

Since node $i$ and $j$ are siblings in the real multicast network, the number of lost probe packets observed at $i$ and $j$ at the same time composes of those lost on the link from the root to their parent node and those lost on both link $i$ and $j$.

$$
n_{i j}^{0}=n\left(1-p_{s}\right)+n p_{s}\left(1-p_{i}\right)\left(1-p_{j}\right)
$$

We denote $\gamma \cdot n_{i j}^{0}$ in Equation (2) by $\Gamma$.

$$
\Gamma=n p_{s}\left(1-p_{i}\right)\left(1-p_{j}\right)=n_{i j}^{0}-n\left(1-p_{s}\right)
$$

$n_{i j}^{0}$ can be obtained by the following equation.

$$
n_{i j}^{0}=n-\left(n_{i}^{1}+n_{j}^{1}-n_{i j}^{1}\right)
$$

$p_{s}$ can be estimated by $\left(n_{i}^{1} \cdot n_{j}^{1}\right) /\left(n \cdot n_{i j}^{1}\right)$ according to Lemma 1.

Then, we derive the following formula for $\Gamma$.

$$
\Gamma=\frac{n_{i}^{1} \cdot n_{j}^{1}}{n_{i j}^{1}}-\left(n_{i}^{1}+n_{j}^{1}-n_{i j}^{1}\right)
$$

Thus, replacing $\Gamma$ in Equation (2) with Equation (8), we get the following equation to estimate $p_{i}$.

$$
\hat{p}_{i}=\frac{n_{i}^{1}}{n_{i}^{1}+n_{j}^{1}-n_{i j}^{1}+\Gamma}=\frac{n_{i j}^{1}}{n_{j}^{1}}=\frac{\sum_{k=1}^{n} X_{i}^{(k)} \wedge X_{j}^{(k)}}{\sum_{k=1}^{n} X_{j}^{(k)}}
$$

Likewise, $p_{j}$ can be estimated as follows.

$$
\hat{p}_{j}=\frac{n_{i j}^{1}}{n_{i}^{1}}=\frac{\sum_{k=1}^{n} X_{i}^{(k)} \wedge X_{j}^{(k)}}{\sum_{k=1}^{n} X_{i}^{(k)}}
$$

Thus, loss rates of the link $i$ and $j$ can be determined as Lemma 2 shows. \#

When $n$ increases to infinity,

$$
\begin{gathered}
\lim _{n \rightarrow \infty} n_{i j}^{1} / n=p_{s} \cdot p_{i} \cdot p_{j} \\
\lim _{n \rightarrow \infty} n_{j}^{1} / n=p_{s} \cdot p_{j}
\end{gathered}
$$

Then, $\lim _{n \rightarrow \infty} \hat{p}_{i}=p_{i}$.

Therefore, it can be concluded that the loss rate estimated by Lemma 2 is consistent with the real loss rate for each link as the number of probe packets goes to infinity. 


\subsection{Algorithm on loss inference}

Since we have deduced the formula on the loss rate of a link with the prior knowledge of the sequence maintained by the node, we can infer loss performance of all the links in a multicast network. In the procedure of topology inference as we presented recently in [9], the " $0-1$ " sequences for each node including those internal nodes have been obtained. So it becomes convenient to infer the link loss rate with the help of our deduced result. The following algorithm infer not only the topology but also loss rate for all the links in the network.

1. Input: The set of receivers $R$, number of probe packets $n$, observed sequences at receivers $\left(X_{k}^{(i)}\right)_{k \in R}^{i=1, \cdots, n}$;

2. $R^{\prime}:=R, V^{\prime}:=R, L^{\prime}:=\phi, h=\max _{k \in R}(k . h o p)$,

$W_{m}=\phi, m=1, \cdots, h ; / / W_{m}$ is a set of nodes with hop count value $m, m$ is initialized as the maximum value of hop count $h$ for all nodes in $R . / /$

3. for $k \in R$, do

4. for $i=1, \cdots, m$ do

5. $\quad$ if $(k . h o p=i)$ then $W_{i}=W_{i} \bigcup\{k\}$;

6. Compute hamming distance $\left(H_{d}(p, q)\right)$ of each pair in $W_{i}$, $H_{d}(p, q)=\sum_{j=1}^{n} X_{p}^{(j)} \otimes X_{q}^{(j)} ;$

7. while $m>1$ do

8. while $W_{m} \neq \phi$ do

9. $\quad \operatorname{search} u_{q, w_{m}}$ to minimize $H_{d}\left(u_{p, w_{m}}, u_{q, w_{m}}\right)$;

10. $\quad$ if $H_{d}\left(u_{p, w_{m}}, u_{q, w_{m}}\right)>\delta_{m}$ then $U=u_{p, w_{m}}$; $/ / \delta_{m}$ is an experienced threshold to compare correlation degree of a pair of nodes denoted by hamming distance//

11. for $i=1$ to $n$

$$
\begin{aligned}
& \text { Calculate } n_{u_{p, w_{m}}}^{1}=\sum_{i=1}^{n} X^{(i)}\left(u_{p, w_{m}}\right), \\
& n_{u_{q, w_{m}}}^{1}=\sum_{i=1}^{n} X^{(i)}\left(u_{q, w_{m}}\right), \\
& n_{u_{p, w_{m}} u_{q, w_{m}}}^{1}=\sum_{i=1}^{n} X^{(i)}\left(u_{p, w_{m}}\right) . \\
& X^{(i)}\left(u_{q, w_{m}}\right) ;
\end{aligned}
$$

12. Calculate loss rates of link $u_{p, w_{m}}$ and $u_{q, w_{m}}$,

$$
\begin{aligned}
& \hat{\alpha}_{u_{p, w_{m}}}=1-\frac{n_{u_{p, w_{m}} u_{q, w_{m}}}^{1}}{n_{u_{q, w_{m}}}^{1}}, \\
& \hat{\alpha}_{u_{q, w_{m}}}=1-\frac{n_{u_{p, w_{m} u_{q, w_{m}}}}^{1}}{n_{u_{p, w_{m}}}^{1}}
\end{aligned}
$$

13. replace $u_{p, w_{m}}, u_{q, w_{m}}$ with $U$; // $U$ is the parent node of $u_{p, w_{m}}, u_{q, w_{m}} / /$

14. for $i=1, \cdots, n$ do $X_{U}^{(i)}:=\vee_{u \in U} X_{u}^{(i)}$;

15. U.hop $:=m-1$;
16.

$$
\begin{aligned}
& V^{\prime}:=V^{\prime} \bigcup\{U\} ; W_{m}:=W_{m} \backslash U ; \\
& W_{m-1}:=W_{m-1} \bigcup U ;
\end{aligned}
$$

17. for each $u \in U$ do $L^{\prime}:=L^{\prime} \bigcup\{(U, u)\}$;

18. $\mathrm{m}:=\mathrm{m}-1$;

19. $V^{\prime}:=V^{\prime} \bigcup\{0\} ; L^{\prime}:=L^{\prime} \bigcup\{0, U\}$;

20. Output: Inferred topology and loss rate $\left(V^{\prime}, L^{\prime}, \hat{\alpha}\right)$

Firstly all the receivers are classified into different node sets $W_{m}(1 \leq m \leq h)$ according to their values of hop count. The hamming distances of each node pair in $W_{m}$ are calculated. Inference begins with identifying siblings in the node set with maximum value of hop count. Calculate the loss rate of the links connected to the identified siblings. Then replace the siblings with their parent node and add the parent node into the node set with hop count reduced by 1 . The " $0-1$ " sequence of the parent node is obtained by "OR" operation of those of the siblings. When all nodes in $W_{m}$ are grouped decrease hop count value by 1 . Repeat the same procedure among the nodes in the node set $W_{m-1}$. The algorithm ends until the hop count becomes 1 . After all, the topology and loss rates of the inferred links are obtained.

\section{Extension to General Trees}

The approach to infer loss performance is extended to general trees in the section, which requires priori knowledge of the inferred topology too. Section 3.2 proposes the algorithm that can infer the network topology and link loss rate simultaneously. So we build a scheme as loss rate-based topology inference where the procedure of topology inference can benefit from the loss rate inferred by our approach.

\subsection{Link loss inference for general trees}

Similarly to the deduction of Lemma 2, we estimate the link loss rate of general trees by Lemma 3.

Lemma 3 The loss rate of link $s_{1}$ can be estimated by $\hat{\alpha}_{s_{1}}$.

$$
\hat{\alpha}_{s_{1}}=1-\hat{p}_{s_{1}}=1-\frac{n_{s_{1} s_{2} s_{3} \ldots s_{m}}^{1}}{n_{s_{2} s_{3} \ldots s_{m}}^{1}}
$$

Where $s_{1}, s_{2}, \ldots, s_{m}$ are siblings.

\section{Proof}

As $n$ increases to infinity,

$$
\lim _{n \rightarrow \infty} n_{s_{1} s_{2} s_{3}}^{1} / n=p_{s} \cdot p_{s_{1}} \cdot p_{s_{2}} \cdot p_{s_{3}}
$$




$$
\lim _{n \rightarrow \infty} n_{s_{2} s_{3}}^{1} / n=p_{s} \cdot p_{s_{2}} \cdot p_{s_{3}}
$$

Thus, $\lim _{n \rightarrow \infty} \frac{n_{s_{1} s_{2} s_{3}}^{1}}{n_{s_{2} s_{3}}^{1}}=\lim _{n \rightarrow \infty} \hat{p}_{i}=p_{i} \sharp$

As the number of probe packets go to infinity, the loss rate estimated by $\hat{p}_{i}$ is consistent with the real loss rate for each link in general trees.

\subsection{Loss rate-based topology inference}

A parameter $\epsilon$ is introduced into grouping siblings in general trees as discussed in $[6,9]$, which requires $\epsilon$ to be less than all internal link loss rates such that the inferred topology can be correctly convergent to the true topology. However, the internal link loss rates are unknown in advance, which causes the wrongly inferred topology with only the high loss rate links such as the discussion about failure inference in [6].

Thus, with the help of the approach to loss rate inference we proposed above, a scheme is built to adjust $\epsilon$ timely based on inferred loss rates.

The grouping step for topology inference starts by finding a binary set $\left\{u_{1}, u_{2}\right\}$ of minimal hamming distance $H_{D}(\cdot, \cdot)$ as defined in [9], then adjoining further elements to it provided any other node pair $\left\{u_{x}, u_{y}\right\}$ satisfies the inequality (10).

$$
H_{d}\left(u_{x}, u_{y}\right)(1-\epsilon)<H_{d}\left(u_{1}, u_{2}\right)
$$

In the procedure of grouping siblings, loss rates of the links are computed. But wrong grouping may exist due to inappropriate choice of $\epsilon$. So when the minimal loss rate among all the links is less than $\epsilon$, we change the value of $\epsilon$ to be the minimal loss rate, and group the siblings again according to the modified $\epsilon$. The procedure is described as follows.

1. Initiate $\epsilon, \hat{\alpha}_{\min }$.

2. While $\hat{\alpha}_{\min }<\epsilon$ do

3. $\epsilon=\hat{\alpha}_{\text {min }}$

4. Group siblings according to inequality (10).

5. Compute the loss rates based on inferred topology, denote the minimized loss rate as $\hat{\alpha}_{\text {min }}$.

$\epsilon$ is initiated to be less than $\hat{\alpha}_{\text {min }}$ in the adjusting procedure, both with quite large values. So some pseud siblings are grouped firstly, which will result in large loss rates. Among them the minimal loss rate is nevertheless, less than the initiated value. Then all the nodes will be grouped again with smaller $\epsilon$, some pseud siblings are removed in this case, which consequently diminishes the minimal loss rate inferred in the newly grouped tree. The nodes are grouped again due to $\hat{\alpha}_{\min }<\epsilon$. The procedure is repeated until $\epsilon$ is adjusted to the appropriate value that satisfies the condition $\epsilon$ is smaller than the loss rates of all links in the network. Thus, the inferred topology can be quickly convergent to the true topology with the appropriate $\epsilon$. Lemma 4 demonstrates the relationship between $\epsilon$ and the link loss rate.

Lemma 4 A large $\epsilon$ causes more pseud siblings grouped in the inferred tree, which makes the loss rates of the links in the network increased. On the other hand, a small $\epsilon$ can remove some pseud siblings thus result in decreased loss rates of the links.

\section{Proof}

Assume that $s_{1}$ is identified to have $m$ siblings including itself and some likely pseud siblings. Then, let $\check{p}_{s_{1}}(m)$ denotes the successfully accepting probability of link $s_{1}$ under the assumption of $m$ siblings.

$$
\check{p}_{s_{1}}(m)=\frac{n_{s_{1} s_{2} s_{3} \ldots s_{m}}^{1}}{n_{s_{2} s_{3} \ldots s_{m}}^{1}}
$$

If a smaller $\epsilon$ causes a node removed into its siblings set according to the inequality (10).

$$
\check{p}_{s_{1}}(m-1)=\frac{n_{s_{1} s_{2} s_{3} \ldots s_{m-1}}^{1}+x}{n_{s_{2} s_{3} \ldots s_{m-1}}^{1}+y}
$$

Both $x$ and $y$ are integer. $x$ is less than $y$ due to the removing of the node whose " 0 - 1 " sequence is much different from those of other nodes. The hamming distance between the sequences maintained by the removing node and other nodes is far from those among other siblings, which leads $x$ to be less than $y$, and both are more than 0 .

Thus,

$$
\check{p}_{s_{1}}(m-1)<\check{p}_{s_{1}}(m)
$$

So a smaller $\epsilon$ leads the adjusting procedure to remove some pseud siblings thus result in decreased loss rates of the links. $\sharp$

The loss rate-based topology inference can thus be more accurate by adjusting $\epsilon$ than those inferred in [6,9]. 


\section{Conclusion}

A novel approach to inferring the network internal loss performance has been proposed in this paper. Incorporating with the topology inference procedure we proposed recently, we have presented an algorithm that infers link loss rate and multicast topology simultaneously. It has been proved that the estimated loss performance by our approach is consistent with the real loss performance in the multicast network. Moreover, we have extended the link loss rate inference to general trees. Based on inferred loss rate, a scheme for inferring correctly convergent topology has been built.

\section{Acknowledgement}

This work was supported by Japan Society for the Promotion of Science (JSPS) Grant-in-Aid for Scientific Research under its General Research Scheme (B) Grant No. 14380139.

\section{References}

[1] R. Caceres, N. G. Duffield, J. Horowitz, and D. Towsley. Multicast-based inference of network-internal loss characteristics. IEEE Trans. Information Theory, 1999.

[2] R. Careres, N. G. Duffield, H. Horowitz, and D. Towsley. Statistical inference for internal link parameters in a network. In Proc. Of the 1998 Annual Meeting of the American Statistical Association Annual Meeting, Dallas, TX, 1998.

[3] R. Careres, N. G. Duffield, H. Horowitz, D. Towsley, and T. Bu. Multicast-based inference of network internal loss characteristics: Accuracy of packet loss estimation. In Proc. Of Infocom'99. New York, NY, 1999.

[4] N. Duffield, J. Horowitz, F. Presti, and D. Towsley. Multicast topology inference from end-to-end measurements. Technical report, ITC Seminar on IP Traffic Measurement, Modeling and Management, Monterey, CA, Sept. 2000.

[5] N. Duffield, J. Horowitz, and F. L. Presti. Adaptive multicast topology inference. In IEEE Infocom 2001, Anchorage, Alaska, April 22-26, 2001.

[6] N. G. Duffield, J. Horowitz, F. L. Presti, and D. Towsley. Multicast topology inference from measured end-to-end loss. IEEE Trans. Information Theory, 2002.

[7] N. G. Duffield and F. F. Presti. Multicast inference of packet delay variance at interior network links. In Pro. IEEE Infocom 2000, Tel Aviv, 2002.
[8] F. L. Presti, N. G. Duffield, J. Horowitz, and D. Towsley. Multicast-based inference of network-internal delay distributions. IEEE/ACM Transactions on Networking, Vol. 10, No. 6, Dec. 2002.

[9] H. Tian and H. Shen. Hamming distance based multicast network topology inference from end-to-end measurements. Submitted for publication. 